\title{
SURFACE EXTENDED ELECTRON LOSS FINE STRUCTURE: DEPENDENCE ON INCIDENT ELECTRON ENERGY AND COLLECTION SOLID ANGLE *
}

\author{
Y.U. IDZERDA **, Ellen D. WILLIAMS ***, T.L. EINSTEIN and \\ Robert L. PARK \\ Department of Physics and Astronomy, University of Maryland, College Park, Maryland 20742, USA
}

Received 7 November 1984; accepted for publication 9 April 1985

\begin{abstract}
Reflected electron energy loss fine structure above the $\mathrm{M}_{2.3}$ core excitation edge of $\mathrm{Cu}$ is measured using two dissimilar electron detection systems, a cylindrical mirror analyzer and an hemispherical grid detector, at primary energies ranging from 700 to $2000 \mathrm{eV}$. We find slight differences in the loss structures measured by the two detector systems, but the radial and pseudo-radial distribution functions calculated by an EXAFS type analysis of the extended fine structure show agreement in the first peak position to within $\pm 0.02 \AA$ for all primary energies above $800 \mathrm{eV}$. Core-loss and fine-structure signals from sub-monolayers of $\mathrm{C}$ and $\mathrm{O}$ were observed. No fine structure was measurable for core-loss signals of single monolayer coverages of $\mathrm{Na}, \mathrm{K}, \mathrm{Cs}$, or $\mathrm{S}$.
\end{abstract}

\section{Introduction}

In the past decade, the measurement of X-ray absorption fine structure above an excitation edge (EXAFS) to determine local bond lengths, atomic types, coordination numbers, and vibrational parameters has earned wide acceptance. For nearly as long, it has been recognized that the mechanism for the creation of the core excitations may not be fundamentally important to the fine-structure measurement [1]. It was suggested that in order to simplify the experimental apparatus, electron bombardment could be used as the excitation mechanism. Work on high-energy, small-angle electron transmission (EXELFS) through thin samples [2] as well as measurements of extended appearance potential fine structure (EAPFS) [3] and Auger-monitored extended fine structure (AMEFS) [4] demonstrated the use of electron bombardment as the electron excitation mechanism.

* From a dissertation to be submitted to the Graduate School, University of Maryland, by Y.U. Idzerda, in partial fulfillment of the requirements for the $\mathrm{PhD}$ degree in physics.

** Maryland Sigma Xi Research Excellence Award recipient.

*** National Science Foundation Presidential Young Investigator. 
A reflection-mode electron scattering analog to EXAFS has recently been suggested by De Crescenzi et al. [5]. As with EAPFS and AMEFS, this electron scattering method not only simplifies the experimental apparatus, but because both the incident and scattered electron beams have relatively low energies $(500-2000 \mathrm{eV})$, the technique is surface sensitive [6]. Unlike EAPFS and AMEFS, in this method the incident electron energy is fixed and troublesome diffraction effects are thus avoided. De Crescenzi et al. have applied this surface extended electron loss fine structure (SEELFS) measurement to a number of substrate and adsorbate systems in an attempt to verify its usefulness [7-10]. Although they have found success in its application, questions have been raised concerning distortion of the fine-structure spectrum due to angular dependence of the fine structure, multiple-loss processes, interference from other edges, as well as analysis distortions from $\mathbf{M}$ edge phase shifts, non-dipole transition processes, and the use of second-derivative data [11-13]. To examine some of the questions of angular dependence, we have measured the extended fine structure (EFS) of the $\mathrm{Cu} \mathrm{M}_{2.3}$ edge in the SEELFS mode using two different detectors, one a cylindrical mirror analyzer (CMA) with an acceptance slit at $42^{\circ}$ from the surface normal, and the second an hemispherical grid analyzer (HGA) with a $2 \pi$ detector solid angle. Measurements were made at various primary energies to display any incident energy dependence. In addition, we have compared the results of directly analyzing data in the first- and second-derivative forms with data integrated to obtain $N(E)$.

\section{Experimental}

The experiments were carried out in an ion-pumped UHV system with base pressure of $1 \times 10^{-10}$ Torr. The system is equipped with a CMA with a coaxial electron gun, an HGA with a phosphor screen for LEED and a coaxial electron gun, a mass spectrometer, an argon-ion sputtering source, and sources for gas and metal deposition. The CMA was used for Auger electron spectroscopy (AES) and electron energy loss spectroscopy (EELS). The HGA was used for EELS and LEED. Lock-in detection was used in AES and EELS. The modulation voltage was applied at the detector for both AES and EELS. The experiments were performed on a single-crystal $\mathrm{Cu}$ sample cut to expose the (111) plane. The sample was cleaned by repeated cycles of Ar-ion bombardment and annealing in vacuum at $500^{\circ} \mathrm{C}$. The major contaminant removed was carbon. Ultimate cleanliness was less than $1 \%$ of any impurity as determined by AES.

SEELFS measurements were taken at primary energies ranging from 700 to $2000 \mathrm{eV}\left(E_{\mathrm{p}}=700,800,1000,1300,1500,1700\right.$, and $\left.2000 \mathrm{eV}\right)$ in the first-derivative and second-derivative modes for the CMA and first-derivative mode for the hemispherical grid analyzer. Typical incident beam currents were $1 \mu \mathrm{A}$ 
for the HGA and $5 \mu \mathrm{A}$ for the CMA. Primary energies were selected to exclude any Auger transition in the measured loss region. Below $700 \mathrm{eV}$, SEELFS signals were present, but reduction of signal made EFS analysis difficult. The measured instrument resolution of $10 \mathrm{eV}$ for all primary energies (except at $2000 \mathrm{eV}$ for the CMA) was defined by the $10 \mathrm{~V} \mathrm{p}-\mathrm{p}$ sinusoidal signal used for lock-in detection of first-derivative spectra for the HGA, and the $5.7 \mathrm{~V} \mathrm{p}-\mathrm{p}$ signal used for the first- and second-derivative spectra of the CMA. At $2000 \mathrm{eV}$ primary energy, the instrument resolution of the CMA was measured to be 12 eV.

To minimize background variation (which greatly facilitates and improves later analysis), special care was taken to properly orient the sample at the focus of the electron optics by monitoring the position and resolution of the elastic peak. Further reduction in background variation was accomplished by optimizing the electron beam focusing conditions to give the flattest background in the measurement range.

\section{Results}

The SEELFS of the $\mathrm{Cu} \mathrm{M}{ }_{2.3}$ core excitation measured in the first-derivative electron distribution $N^{\prime}(E)$ mode at different primary energies, can be seen in figs. 1 and 2. Typically, 10 scans (each covering a range of $80-400 \mathrm{eV}$ of loss energy beyond the $\mathrm{Cu} \mathrm{M}_{2,3}$ core-loss feature at $74 \mathrm{eV}$ and requiring 30 $\mathrm{min} / \mathrm{scan}$ ) were summed to reduce noise. The EFS spectra are very similar for all primary energies. (Due to background differences created by differing detector types, direct comparison between the HGA and CMA raw spectra can be misleading.) After proper background subtraction (described below), we find that the HGA spectra have twice the signal-to-noise ratio, but are otherwise essentially identical to the CMA spectra. The changing peak at 115 $\mathrm{eV}$ loss energy indicated by the arrow, apparent in the HGA spectra and present but masked by the background variation in the CMA spectra, corresponds to the $\mathrm{Cu} \mathrm{M}$ ionization feature. As the incident energy decreases, the $M_{1}$ ionization peak increases while all other features remain essentially unchanged. The presence of the $\mathrm{Cu} \mathrm{M}$ ionization peak in the measured loss region, coupled with the quick decay of the EFS at higher loss energies, yields a rather limited data range of $120-400 \mathrm{eV}$ in loss energy. These energies correspond to a limited $k$ range of $3-9 \AA^{-1}$, a range comparable to that in SEXAFS but less than EAPFS or AMEFS.

The measured extended fine structure was analyzed using analysis programs developed at the University of Maryland [4]. To obtain the undifferentiated electron distribution $N(E)$, a straight-line background was subtracted from the first-derivative data, the resulting curve was integrated and a two-section cubic-spline was subtracted. The data were converted from energy to wave- 


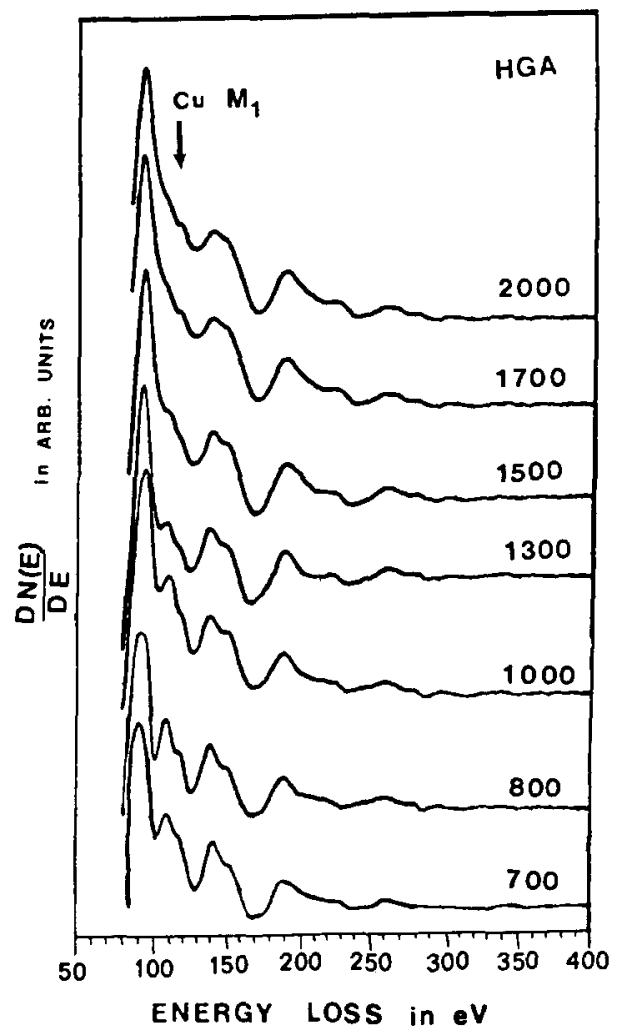

Fig. 1. The first derivative of the electron distribution for incident energies $700-2000 \mathrm{eV}$ as a function of energy loss for $\mathrm{Cu}(111)$ as measured by an HGA electron detector. To emphasize the extended fine structure, we have omitted the elastic peak at $E_{\text {loss }}=0 \mathrm{eV}$ and the $\mathbf{M}_{2,3}$ core-loss features at $E_{\text {loss }}=74 \mathrm{eV}$.

number dependence by choosing an appropriate value for the zero of energy [14]. We used $E_{0}=75 \mathrm{eV}$ in loss energy, which roughly corresponds to the $\mathrm{Cu}$ $\mathrm{M}_{2,3}$ ionization energy. Using values for $E_{0}$ ranging from 65 to $85 \mathrm{eV}$ caused the first peak position of the Fourier transform of the data to shift [15], but the agreement of the first peak position for the different measurements remained the same.

The backscattering amplitude function for $\mathrm{Cu}$ is actually an increasing function of $k$ over the lower half of our $k$ range (from 3-6 $\AA^{-1}$ ) [16,17], in sharp contrast with its asymptotic $k^{-2}$ decrease at high $k$. For this reason, the $\chi(k)$ data were weighted by a single power of $k$ (rather than $k^{3}$ ) to counteract the explicit $k^{-1}$ dependence of $\chi(k)$. The weighted $\chi(k)$ data were Fourier transformed from $k$ (wave number) space to $R$ (distance) space to arrive at the radial distribution function (RDF). Although weighting the $\chi(k)$ data by $k$ is 


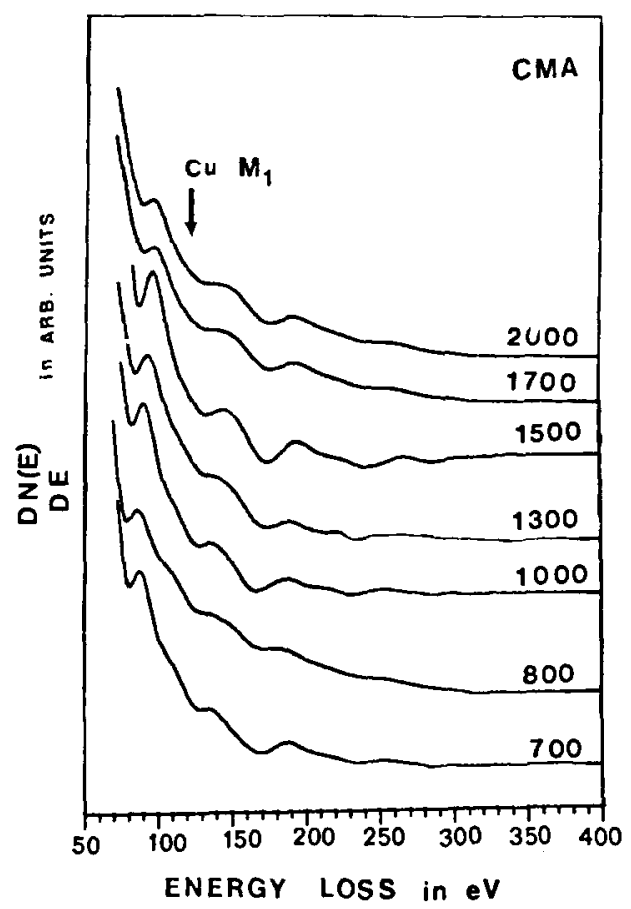

Fig. 2. The first derivative of the electron distribution for incident energies $700-2000 \mathrm{eV}$ as a function of energy loss for $\mathrm{Cu}(111)$ as measured by a CMA electron detector. To emphasize the extended fine structure, we have omitted the elastic peak at $E_{\text {loss }}=0 \mathrm{eV}$ and the $\mathbf{M}_{2,3}$ core-loss feature at $E_{\text {loss }}=74 \mathrm{eV}$.

preferred from theoretical considerations, $k^{2}$ weighting was also used with no change in the first peak agreement. Both $k$ and $k^{2}$ weighting gave roughly flat weighted $\chi(k)$ spectra to equally emphasize low- and high- $k$ information. The weighted $\chi(k)$ data, derived from the integrated $N(E)$ data as measured by CMA and HGA, are shown in figs. 3 and 4. It is apparent that the spectra do not depend very much at all on $E_{\mathrm{p}}$ or detection angle.

Since our major concern is with these dependencies, we have not included phase shift information in the Fourier transform. Thus our analysis and conclusions avoid questions regarding $\mathrm{M}$-edge phase shifts and possible nondipole transitions. While these concerns are important to understanding the detailed mechanism of SEELFS, they initially may be circumvented by the use of suitable standards - if they can be found - for direct comparison, as suggested by Stern [18]. Omitting the phase shift results in calculated radial distribution functions (RDFs) which do not represent the actual copper atomic neighboring distances but which can be internally compared to show data similarity. 

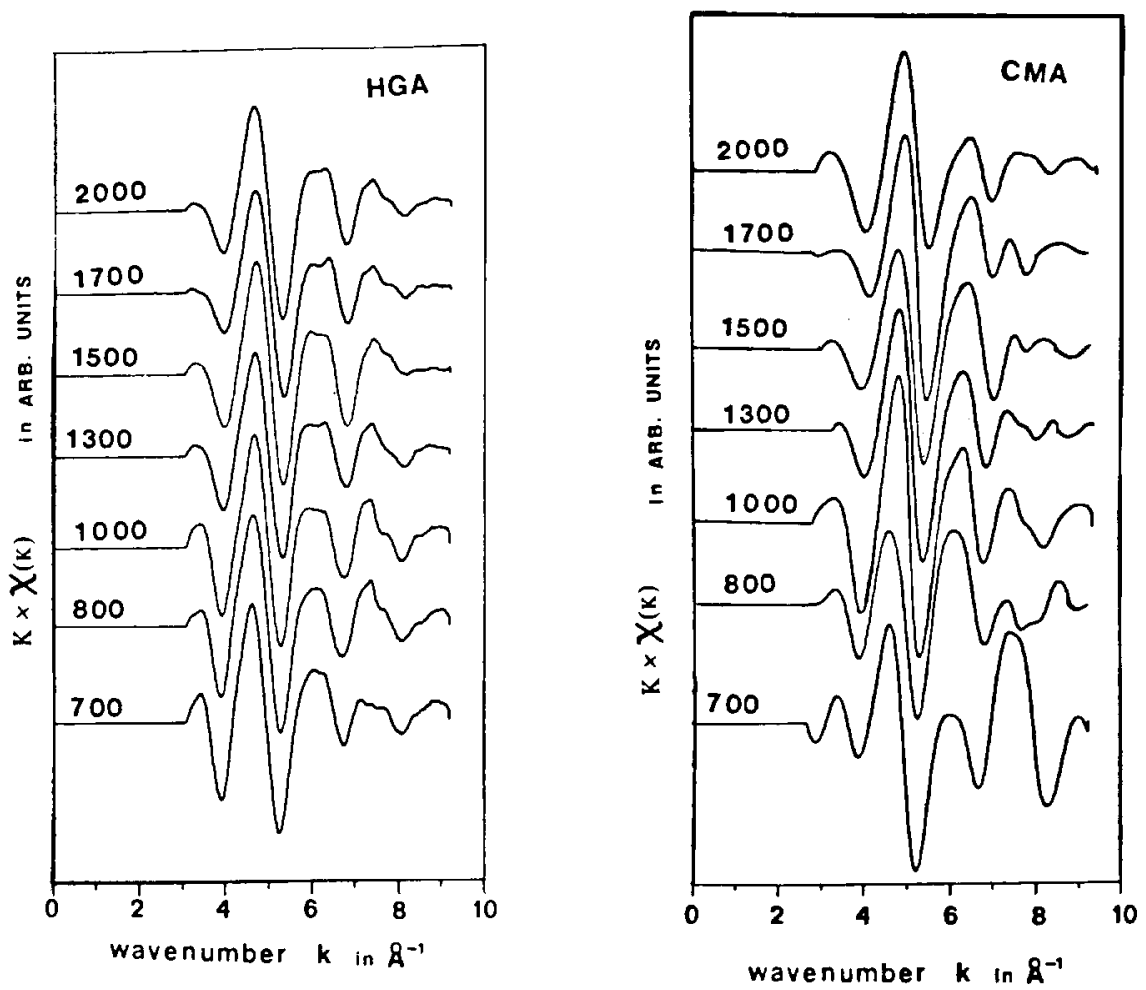

Fig. 3. Integrated $N(E)$ data weighted by a factor of $k$ for incident electron energies $700-2000 \mathrm{eV}$ measured by HGA detector.

Fig. 4. Integrated $N(E)$ data weighted by a factor of $k$ for incident electron energies $700-2000 \mathrm{eV}$ measured by CMA detector.

The RDFs for the integrated CMA and HGA data are shown in figs. 5 and 6. The agreement between the RDFs is an indication of both the similarity of the original data and the success of the background subtraction. Any low- $R$ peaks $(\leqslant 1.5 \AA)$ are artifacts of the background subtraction and of truncation errors. These peaks vary in magnitude and position as the background subtraction parameters are varied. The higher- $R$ peaks do not change significantly in position or magnitude as the background subtraction parameters are varied and would represent nearest-neighbor distances if the phase shifts were included in the Fourier transform. The calculated first peak positions as a function of primary beam energy and detector type are listed in table 1 . The agreement for the first peak position is $\pm 0.02 \AA$. A more accurate way to determine differences in radial distances is the ratio method, developed for comparing unknown coordination distances with a standard sample of known 

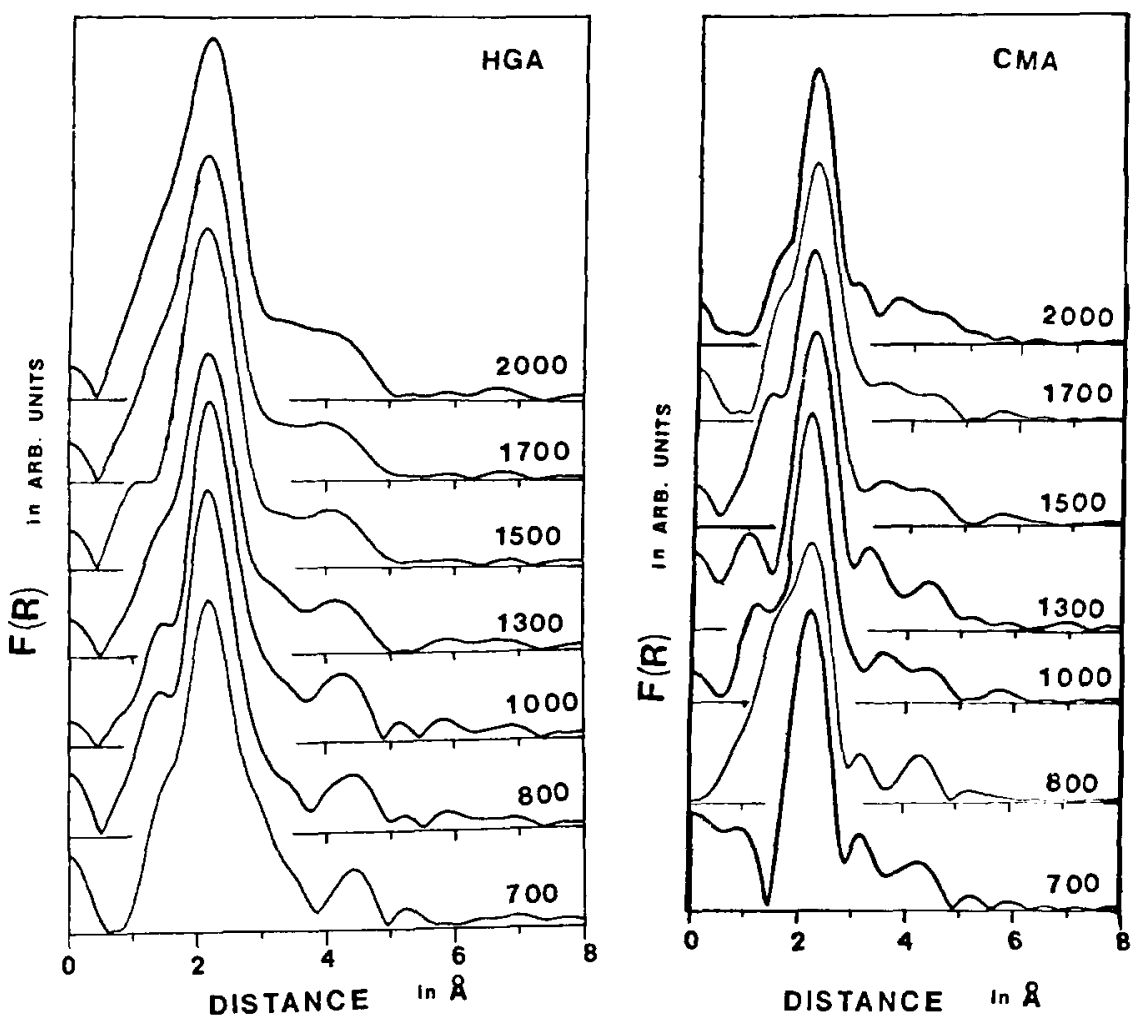

Fig. 5. Fourier transforms of the intergrated $N(E)$ data for copper showing radial positions of neighboring copper atoms for incident energies $700-2000 \mathrm{eV}$ as measured by an HGA.

Fig. 6. Fourier transforms of the integrated $N(E)$ data for copper showing radial positions of neighboring copper atoms for incident energies $700-2000 \mathrm{eV}$ as measured by a CMA.

coordination distance [18]. This method, when applied to the different spectra resulted in the same agreement in peak position, as indicated above. Considering the limited $k$ range, this agreement is very good. Examination of the figures shows that the HGA gave stronger EFS and weaker background variation than the CMA. This results in more accurate background subtraction and thus RDFs that have weaker artificial low- $R$ peaks (artifacts of incomplete background subtraction) and so are presumably more accurate.

The first-derivative $N^{\prime}(E)$ and second-derivative $N^{\prime \prime}(E)$ spectra were analyzed similarly. For the $N^{\prime}(E)$ data, we removed a two-section cubic-spline background, converted to $\chi^{\prime}(k)$ data (again $E_{0}=65-85 \mathrm{eV}$ gave acceptable results; we chose $E_{0}=74 \mathrm{eV}$ ), weighted by a factor of $k^{2}$, and Fourier transformed to get a radial distribution function different from the RDF 
Table 1

First peak position (in $\AA$ ) of the radial distribution functions for integrated $N(E)$ data and of the pseudo-radial distribution functions for the first- and second-derivative data for incident energies $700-2000 \mathrm{eV}$ and HGA and CMA detector systems

\begin{tabular}{|c|c|c|c|c|c|c|}
\hline \multirow{2}{*}{$\begin{array}{l}\text { Incident } \\
\text { energy } \\
(\mathrm{eV})\end{array}$} & \multicolumn{2}{|c|}{$N(E)$} & \multicolumn{2}{|c|}{$N^{\prime}(E)$} & \multicolumn{2}{|c|}{$N^{\prime \prime}(E)$} \\
\hline & CMA & $\overline{\mathrm{HGA}}$ & $\overline{C M A}$ & $\overline{\mathrm{HGA}}$ & $\overline{\mathrm{CMA}}$ & $\overline{\text { HGA }}$ \\
\hline 700 & 2.19 & 2.21 & 2.20 & 2.26 & 2.16 & 2.23 \\
\hline 800 & 2.20 & 2.22 & 2.25 & 2.26 & 2.24 & 2.24 \\
\hline 1000 & 2.19 & 2.22 & 2.25 & 2.28 & 2.30 & 2.26 \\
\hline 1300 & 2.20 & 2.22 & 2.24 & 2.26 & 2.26 & 2.26 \\
\hline 1500 & 2.20 & 2.18 & 2.28 & 2.24 & 2.24 & 2.30 \\
\hline 1700 & 2.21 & 2.18 & 2.25 & 2.25 & 2.25 & 2.28 \\
\hline 2000 & 2.18 & 2.19 & 2.25 & 2.26 & 2.26 & 2.27 \\
\hline \multirow[t]{3}{*}{ Average $^{\text {a) }}$} & 2.20 & 2.20 & 2.26 & 2.26 & 2.27 & 2.27 \\
\hline & \pm 0.02 & \pm 0.02 & \pm 0.02 & \pm 0.02 & \pm 0.03 & \pm 0.03 \\
\hline & \multicolumn{2}{|c|}{$2.20 \pm 0.02$} & \multicolumn{2}{|c|}{$2.26 \pm 0.02$} & \multicolumn{2}{|c|}{$2.27 \pm 0.03$} \\
\hline
\end{tabular}

a) Average does not include $700 \mathrm{eV}$ measurement. Lower primary energies result in lower signal-to-noise level as indicated by the poor agreement of $700 \mathrm{eV}$ data.

arrived at for the integrated $N(E)$ data. (The energy differentiation results in an additional $k^{-1}$ dependence in the $\chi^{\prime}(k)$ data. If $k^{3}$ weighting is used for $\chi^{\prime}(k)$ data, we still get good agreement.) De Crescenzi et al. labeled these as pseudo-radial distribution functions. The pseudo-RDFs are shown in figs. 7 and 8 . The calculated peak position is different from the integrated $N(E)$ data (as would be expected with the omission of the energy-dependent phase shifts), but we found similar agreement of peak position as a function of the various energies and detector systems. The calculated first peak positions are listed in table 1.

The background subtracted $N^{\prime}(E)$ data obtained by the HGA detection system were differentiated using an 11-point Savitzky-Golay method to obtain $N^{\prime \prime}(E)$ data $[19,20]$. These data were converted to $\chi^{\prime \prime}(k)\left(E_{0}=74 \mathrm{eV}\right.$, but may range from 65 to $85 \mathrm{eV}$ ), weighted by $k^{3}$, and Fourier transformed to get a different pseudo-radial distribution function. (A weighting of $k^{4}$ resulted in poor Fourier transforms and bad first peak agreement.) This PRDF was compared to a PRDF obtained from the CMA $N^{\prime \prime}(E)$ measured data (or similarly differentiated $N^{\prime}(E)$ CMA data). (De Crescenzi et al. used CMA measured second-derivative $N^{\prime \prime}(E)$ data for their analysis.) The agreement in first peak position changes to $\pm 0.03 \AA$ for the $N^{\prime \prime}(E)$ data. This poorer agreement is most likely due to the increased apparent noise level at high $k$ due to the effect of the higher- $k$ weighting. After $k$ weighting, the noise level is significantly larger for the weighted $N^{\prime \prime}(E)$ data than for the $N(E)$ or the $N^{\prime}(E)$ data. These first peak positions of the PRDFs are also listed in table 1. 

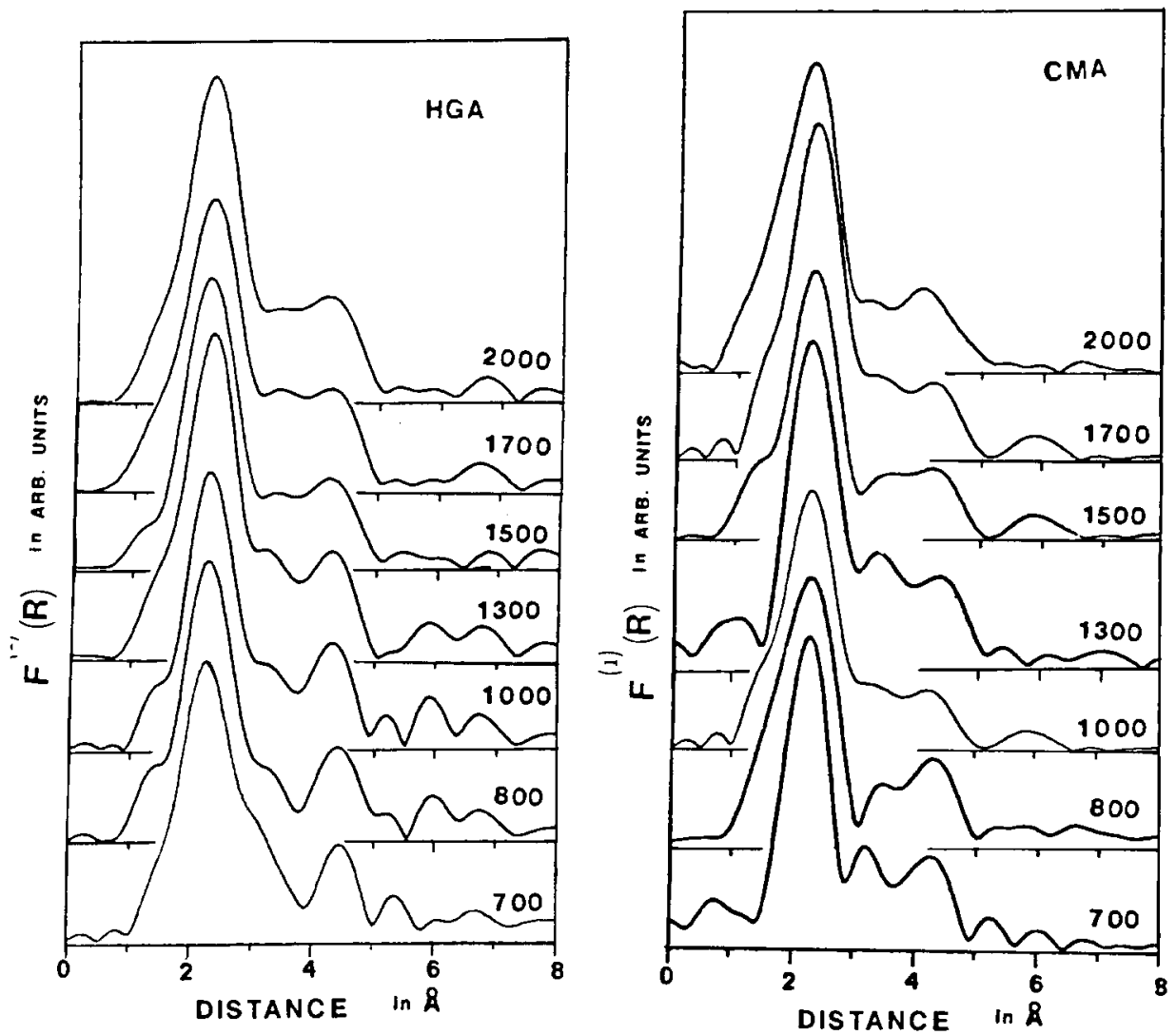

Fig. 7. Fourier transforms of the differentiated $N^{\prime}(E)$ data for copper showing radial positions of neighboring copper atoms for incident energies $700-2000 \mathrm{eV}$ as measured by an HGA.

Fig. 8. Fourier transforms of the differentiated $N^{\prime}(E)$ data for copper showing radial positions of neighboring copper atoms for incident energies $700-2000 \mathrm{eV}$ as measured by a CMA.

Comparison between HGA and CMA measurements using $N(E), N^{\prime}(E)$, or $N^{\prime \prime}(E)$ results shows good agreement for all primary energies greater than $700 \mathrm{eV}$. Examining Fourier transforms shows, as others have maintained [8], that the energy differentiation enhances higher- $R$ peaks. For proper analysis, the selection of the zero of energy using a Lee-Beni type method requires the use of $N(E)$ data [13]. Unfortunately, as the RDFs demonstrate, integrated $N(E)$ data are more sensitive to incomplete background subtraction than the measured $N^{\prime}(E)$ or $N^{\prime \prime}(E)$ data. Thus, use of integrated $N(E)$ data compounds the requirements of background minimization, but is still the preferred data form. It should be noted that comparison of first peak position between 
these three data types can be misleading. Energy differentiation and integration changes the pre-factor $k$-weighting found in the standard EXAFS equation and introduces changes in the phase shifts which must be included in a complete analysis. The limited analysis we have performed, which does not include phase shift information or a proper determination of the zero of energy, does not correct for the alterations to the EFS analysis created by energy differentiation or integration. Thus, it is not surprising that the calculated first peak positions are outside the error bar. What still remains important is the agreement within the data types.

Due to the surface sensitivity of this technique, a major application of the SEELFS method is to adsorbate/substrate systems. (SEELFS for C on Ni(110) $[7,8]$ and $\mathrm{O}$ on $\mathrm{Ni}(100)$ [9] have already been measured by De Crescenzi et al.) As a further test of this method we attempted to measure the SEELFS of single monolayers of $\mathrm{Na}, \mathrm{K}, \mathrm{Cs}$, and $\mathrm{S}$ on the $\mathrm{Cu}(111)$ substrate. The $\mathrm{Na}, \mathrm{K}$, and $\mathrm{Cs}$ overlayers were evaporated onto the $\mathrm{Cu}$ substrate from a source at a rate of 1 monolayer per 15 minutes. The $S$ overlayer was obtained by exposure of the $\mathrm{Cu}$ substrate to $\mathrm{H}_{2} \mathrm{~S}$ gas. Coverages were monitored by Auger spectroscopy. LEED measurements showed ordered overlayers for $\mathrm{K}, \mathrm{Cs}$, and $\mathrm{S}$ and a diffuse substrate pattern for $\mathrm{Na}$. In all cases, small core excitation features ( $\mathrm{K}$ edge of $\mathrm{Na}, \mathrm{L}_{2,3}$ edge of $\mathrm{K}, \mathrm{M}_{4}$ edge of $\mathrm{Cs}$, and $\mathrm{L}_{2,3}$ edge of $\mathrm{S}$ ) were observed, but minimal or no EFS was visible above the noise level. The EFS for the K overlayer (beginning at $300 \mathrm{eV}$ loss energy) was visible, but interference with the $\mathrm{Cu}, \mathrm{M}_{2,3}$ edge EFS (beginning at $\sim 70 \mathrm{eV}$ loss energy) made analysis impossible. Similar measurements on the $\mathrm{K}$ edge of $\mathrm{C}$ and $\mathrm{O}$ sub-monolayer coverages, present before surface cleaning, produced significantly stronger (factor of 3-20) core-loss features with accompanying fine structure. Detailed analyses of these spectra were not performed. These results suggest that the SEELFS method is applicable to low-atomic-number or low-binding-energy sub-monolayer adsorbate systems, in agreement with electron cross-section calculations which show a very rapid decrease as a function of atomic number [21].

\section{Conclusions}

In conclusion, we have measured the surface extended electron loss fine structure (SEELFS) of the $\mathrm{Cu} \mathrm{M}_{2,3}$ edge at primary energies from 700 to 2000 $\mathrm{eV}$ using CMA and HGA electron detectors. We found good similarity in the EFS and excellent agreement $( \pm 0.02 \AA$ for the integrated and first-derivative data and slightly worse agreement for the second-derivative data) in the first peak positions of the radial distribution functions (RDF). This agreement suggests that multiple-loss processes (which are dependent on primary energy) are not present to a significant extent in copper. To determine accurate 
absolute interatomic distances (using phase shifts and energy-zero adjustment), the integrated $N(E)$ data should be analyzed. Since the integrated $N(E)$ data are more sensitive to incomplete background removal, it is important to minimize background variation by proper sample positioning and electron gun focusing. Special care must be taken in performing the background subtraction to obtain good RDFs. We have also found that because the calculated RDFs are so similar for the two detector systems, we anticipate no significant angular dependence to the EFS (except perhaps in signal strength). Measurements on single monolayers of $\mathrm{Na}, \mathrm{K}, \mathrm{Cs}$, and $\mathrm{S}$ showed small core-loss features, but no measurable EFS, suggesting a limited application to monolayer coverages of low-atomic-number or low-binding-energy absorbates.

\section{Acknowledgements}

We would like to thank H. Iwasaki and J. Vahakangas for their contributions to this work. Support was provided by the Department of Energy under grant no. DE-FG05-84ER-45071. One of us (E.D.W.) has been supported by a Summer Research Award from the University of Maryland graduate school. Computer facilities were provided by the Computer Science Center of the University of Maryland.

\section{References}

[1] T.L. Einstein, Appl. Surface Sci. 11/12 (1982) 42, and references therein.

[2] C. Colliex and B. Jouffrey, Phil. Mag. 25 (1972) 491.

[3] T.L. Einstein, M.J. Mehl, J.F. Morar, R.L. Park and G.E. Laramore, in EXAFS and Near Edge Structures, Eds. A. Bianconi, L. Incoccia and S. Stipcich (Springer, Berlin, 1983) p. 391.

[4] J.F. Morar and R.L. Park, J. Vacuum Sci. Technol. A1 (1983) 1043.

[5] M. De Crescenzi, in: EXAFS and Near Edge Structures, Eds. A. Bianconi, L. Incoccia and S. Stipcich (Springer, Berlin, 1983) p. 382.

[6] M. De Crescenzi, G. Chiarello, E. Colavita and R. Rosei, Solid State Commun. 44 (1982) 285.

[7] L. Papagano and L. Caputi, Phys. Rev. B29 (1984) 1483.

[8] R. Rosei, M. De Crescenzi, F. Sette, C. Quaresima, A. Savoia and P. Perfetti, Phys. Rev. B28 (1983) 1161.

[9] M. De Crescenzi, F. Antonangeli, C. Bellini and R. Rosei, Phys. Rev. Letters 50 (1983) 1949.

[10] M. De Crescenzi, G. Chiarello, E. Colavita and R. Memeo, Phys. Rev. B29 (1984) 3730.

[11] A.P. Hitchcock and C.H. Teng, J. Vacuum Sci. Technol. A1 (1983) 1209;

A.P. Hitchcock and C.H. Teng, to be published.

[12] W. Ekardt and D.B. Tran Thoai, Solid State Commun. 45 (1983) 1083.

[13] M.J. Mehl and T.L. Einstein, to be published; M.J. Mehl and T.L. Einstein, Bull. Am. Phys. Soc. 29 (1984) 516.

[14] Normally the Lee-Beni method [P.A. Lee and G. Beni, Phys. Rev. B15 (1982) 282] is used to determine the zero of energy for the Fourier transform, but since no phase shifts are included, such a method is impractical. 
[15] S.P. Hershfield and T.L. Einstein, Phys. Rev. B29 (1984) 1048.

[16] B.K. Teo and P.A. Lee, J. Am. Chem. Soc. 101 (1979) 2815.

[17] B.K. Teo, P.A. Lee, A.L. Simons, P. Eisenberger and B.M. Kincaid, J. Am. Chem. Soc. 99 (1977) 3834.

[18] E.A. Stern, D.E. Sayers and F.W. Lytle, Phys. Rev. B11 (1975) 4836.

[19] A. Savitzky and M. Golay, Anal. Chem. 36 (1964) 1627.

[20] J. Steinier, Y. Termonia and J. Deltour, Anal. Chem. 44 (1972) 1906.

[21] R.D. Leapman, P. Rez and D.F. Mayers, J. Chem. Phys. 72 (1980) 1232. 